\title{
Self-assembly in soft matter with multiple length scales
}

\author{
Alberto Scacchi $\odot,{ }^{1,2,3, *}$ Sousa Javan Nikkhah $\odot,{ }^{2}$ Maria Sammalkorpi $\odot,{ }^{2,4}$ and Tapio Ala-Nissila $\odot^{1,5}$ \\ ${ }^{1}$ Interdisciplinary Centre for Mathematical Modelling and Department of Mathematical Sciences, Loughborough University, \\ Loughborough, Leicestershire LE11 3TU, United Kingdom \\ ${ }^{2}$ Department of Chemistry and Materials Science, Aalto University, P.O. Box 16100, FI-00076 Aalto, Finland \\ ${ }^{3}$ Department of Applied Physics, Aalto University, P.O. Box 11000, FI-00076 Aalto, Finland \\ ${ }^{4}$ Department of Bioproducts and Biosystems, Aalto University, P.O. Box 16100, FI-00076 Aalto, Finland \\ ${ }^{5}$ Quantum Technology Finland Center of Excellence and Department of Applied Physics, \\ Aalto University, P.O. Box 11000, FI-00076 Aalto, Finland
}

(Received 20 January 2021; accepted 25 March 2021; published 23 April 2021)

\begin{abstract}
Spontaneous self-assembly in molecular systems is a fundamental route to both biological and engineered soft matter. Simple micellization, emulsion formation, and polymer mixing are well understood. However, the principles behind emergence of structures with competing length scales in soft matter systems remain unknown. Examples include droplet-inside-droplet assembly in many biomacromolecular systems undergoing liquid-liquid phase separation, analogous multiple emulsion formation in oil-surfactant-water formulations, and polymer core-shell particles with internal structure. We develop here a microscopic theoretical model based on effective interactions between the constituents of a soft matter system to explain self-organization both at single and multiple length scales. The model identifies how spatial ordering at multiple length scales emerges due to competing interactions between the system components, e.g., molecules of different sizes and different chemical properties. As an example of single and multiple length scale assembly, we map out a generic phase diagram for a solution with two solute species differing in their mutual and solvent interactions. We further connect the phase diagram to a molecular system via molecular simulations of a block-copolymer system that has a transition from regular single-core polymer particles to multicore aggregates that exhibit multiple structural length scales. The findings provide guidelines to understanding the length scales rising spontaneously in biological self-assembly but also open venues to the development and engineering of biomolecular and polymeric functional materials and pharmaceutical formulations.
\end{abstract}

DOI: 10.1103/PhysRevResearch.3.L022008

Self-assembly is nature's ingenious route to create materials with complex structural and functional properties. Examples range from biological self-assembly, such as protein assemblies [1], cellular condensates [2], viruses [3], or cell membranes and their internal structure, such as lipid rafts [4,5], to widely used systems emerging from, e.g., polymer self-assembly [6-8], molecular crystals [9,10], or bio-inspired approaches to materials engineering $[1,11]$.

Particularly fascinating self-assembly materials are those exhibiting multiple length scales in their spatial arrangement. Examples include hierarchical biological and bio-inspired materials, such as bone, nacre, or crustacean exoskeletons [5], and silklike materials [12], all featuring exceptional toughness and resilience. Many block-copolymer systems [8], coacervate droplets in biocondensates (liquid-liquid phase separation in biological systems) [2,13], and multiple emulsions [14] readily exhibit hierarchical multiple length scales

\footnotetext{
*alberto.scacchi@aalto.fi

Published by the American Physical Society under the terms of the Creative Commons Attribution 4.0 International license. Further distribution of this work must maintain attribution to the author(s) and the published article's title, journal citation, and DOI.
}

when self-assembling. This associates with locally varying molecular environments in terms of density and confinement, dielectric properties, or hydrophobicity and hydrophilicity, granting access to fascinating applications in nanophotonics $[10,15,16]$, organic electronics $[10,17]$, confined catalysis $[18,19]$, energy materials [11], and sensors [10,16,18]. Another important field of application is pharmaceutical materials and drug delivery $[8,16,18]$, where the heterogenous, often compartmentalized, solvation environment is interesting for sequential delivery of multiple drug species, for instance, in cancer therapy $[20,21]$.

Simple molecular self-organization, such as aqueous micellization and emulsion formation, can be easily explained at the level of the interplay between water entropy, the relevant surface tensions, and the corresponding free energies [22]. Multiple structural length scales emerge in the presence of competing interactions $[8,23]$, yet fundamental theoretical underpinning to deeply understand this is lacking. We present here a theoretical framework demonstrating how competition between molecular interactions can lead to spontaneous formation of structurally complex self-assembly matter exhibiting multiple length scale structural features and demonstrate its connection to a block-copolymer system.

We employ energy minimization principles based on classical density functional theory (DFT) [24-27] to model the 
spatially varying average density of each component in the thermodynamic equilibrium in a multicomponent soft matter system. The interactions are approximated by a free-energy potential. This approach provides a generic phase diagram exhibiting a variety of possible equilibrium assembly configurations as a function of the system composition. We present a formulation that covers both the formation of single length scale, simple phases and a multiple periodic phase. The latter is commensurate with, e.g., formation of droplets inside droplets, such as biomolecular condensates [2], or multicore polymer micelles [28-31].

For computational efficiency, we consider a twodimensional (2D) model system composed of two species in a solvent; however, the formalism is also valid in three dimensions (3D). The system is characterized by soft interactions, where the effective pair potentials between coarse-grained complex molecules are designed using the generalized exponential model of index $n(\mathrm{GEM}-n)$. Such potentials have been extensively used to describe the effective interactions in a vast variety of polymeric systems [32-39]. GEM- $n$ models exhibit interesting pattern formation if they are part of the $Q \pm$ interaction class [40], i.e., for $n>2$. This theory applies generally to any molecular system that has three partially immiscible components. One of the components can be considered as the solvent, implicitly present in the interaction potentials of the two explicit species. Such systems are common in, e.g., aqueous polymer mixtures, biomolecular and colloidal systems, emulsions, and various liquid-crystal-forming systems.

The statistical distribution of the average densities $\rho_{i}(\mathbf{r})$ are obtained using the Ramakrishnan-Yussouff approximation [41] (details in the Supplemental Material (SM) [42]). The fundamental condition for thermal stability of any selfassembled structure is that it corresponds to a minimum in the relevant thermodynamic potential. We choose a mixture of big (b) and small $(s)$ coarse-grained particles interacting via the effective potentials

$$
\begin{aligned}
& \phi_{b b}(r)=\varepsilon_{b b} \exp \left[-\left(\frac{r}{R_{b b}}\right)^{4}\right] ; \quad \phi_{s s}(r)=\varepsilon_{s s} \exp \left[-\left(\frac{r}{R_{s s}}\right)^{8}\right] ; \\
& \phi_{b s}(r)=\varepsilon_{b s} \exp \left[-\left(\frac{r}{R_{b s}}\right)^{2}\right]+\varepsilon_{b s}^{+} \exp \left[-\frac{1}{2} \frac{r}{R_{b s}}\right],
\end{aligned}
$$

where the subscripts refer to the interacting particle pair. $\varepsilon_{b b}>0$ and $\varepsilon_{s s}>0$ represent repulsion between pairs of $b$ and $s$ particles, respectively, corresponding to energy penalty for overlap due to entropic effects. Here, $\varepsilon_{b s}$ imposes an attraction between $b$ and $s$, whereas $r$ is the distance between the centers of the particles. In water solutions, such effective attraction typically arises between hydrophobic components as a result of entropic contributions. It could also be generated by charge distributions on the molecules. Specifically, for charged species such as polyelectrolytes, this interaction can be easily tuned by salt. A long tail repulsion via $\varepsilon_{b s}^{+}>0$ is useful in preventing singularities. Here, $R_{b b}$ and $R_{s s}$ define the sizes of the particles (typically comparable with the radius of gyration for polymers). Similar theoretical approaches have been used to describe other forms of self-assembly, such as superlattice structures [43] and quasicrystals [44].

To choose a relevant set of parameters for Eq. (1), we consider the linear dispersion relation branches $\omega_{ \pm}(k)$, derived in Ref. [45], which characterize the growth or decay of density modulations, with wave number $k$, in the liquid state. The dependence of this quantity with the different parameters in Eq. (1) is rather complicated. Before investigating such a complex quantity, we start by assuming that the mixture is formed by two independent components. This can be ensured by carefully selecting the form of Eq. (1). To have two clearly distinct length scales, we set the ratio $k_{s} / k_{b} \approx 20$, where $k_{s}$ and $k_{b}$ correspond to the large and small particle characteristic wave lengths, respectively. We set $R_{b b}=5$ and $R_{s s}=0.25$, and use dimensionless units throughout. When considering two separate systems of $b$ and $s$ particles, respectively, the linear dispersion relation for each component reads [46-50]

$$
\omega_{i}(k)=-\frac{k^{2} D_{i}}{S_{i}(k)}, \quad i=b, s ;
$$

where $D_{i}$ is the diffusion coefficient and $S_{i}(k)$ the liquid structure factor of component $i$, which can be measured in experiments and is accessible through $S_{i}(k)=1 /\left[1-\rho_{i} \hat{c}_{i}(k)\right]$ [26], where $\hat{c}_{i}(k)$ is the Fourier transform of the direct pair correlation function (see SM [42] for details), and $\rho_{i}$ is the bulk density of species $i$. With this approach, one can easily obtain the values of $\varepsilon_{i i}$ necessary to achieve linear instability for a given critical value of densities $\rho_{i}^{\mathrm{c}}, i=b, s$, and vice versa. It is convenient (but not mandatory) to set $\rho_{b} \approx \rho_{s}$. We choose $\beta \varepsilon_{b b}=0.45$ and $\beta \varepsilon_{s s}=70$, where $\beta=1 /\left(k_{B} T\right), k_{B}$ being the Boltzmann constant and $T$ the temperature. This choice implies that, under the hypothesis of two independent particle systems, the critical densities are $\rho_{b}^{c} \approx 0.55$ and $\rho_{s}^{c} \approx$ 0.78 . These values are obtained by finding the corresponding bulk densities equivalent to the onset of linear instability in Eq. (2), i.e., $\omega_{i}(k)=0$ and $d \omega_{i}(k) / d k=0$. A common choice for the cross-interaction radius is $R_{b s}=\frac{1}{2}\left(R_{b b}+R_{s s}\right) \approx 3$. To avoid a singularity in the density distribution, one should restrict to $2 \pi \int_{0}^{\infty} d r r \phi_{b s}(r)>0$. However, negative values of the integrated strength can be compensated by the strength of repulsion arising from $\phi_{b b}(r)$ and $\phi_{s s}(r)$. Furthermore, the attractive part of $\phi_{b s}(r)$ should be strong enough to favor mixing, and the repulsive part must be small enough to avoid phase separation. Here, we choose $\beta \varepsilon_{b s}=-0.45$ and $\beta \varepsilon_{b s}^{+}=$ 0.02. Designing Eq. (1) with the conditions provided above allows us to have a mixture in which $\rho_{i}^{c}$ does not influence $\rho_{j}^{c}$, for $i \neq j$. This property is evident in the fact that the instability lines in the phase diagram of Fig. 1 (to be explained below) are almost vertical and horizontal, respectively. This condition is not required; however, it is very helpful in avoiding complications due to the interplay of $\phi_{b s}$ in $\omega_{ \pm}(k)$. In fact, under some conditions, this interplay could, e.g., partially or fully suppress one or both instabilities. The model parameters used in Eq. (1) are summarized in Table I. We emphasize that results like those obtained here are expected for different parameter sets, but also for models different from Eq. (1).

TABLE I. Set of dimensionless parameters used in Eq. (1).

\begin{tabular}{ccccccc}
\hline \hline$\beta \varepsilon_{b b}$ & $\beta \varepsilon_{b s}$ & $\beta \varepsilon_{b s}^{+}$ & $\beta \varepsilon_{s s}$ & $R_{b b}$ & $R_{b s}$ & $R_{s s}$ \\
\hline 0.45 & -0.45 & 0.02 & 70 & 5 & 3 & 0.25 \\
\hline \hline
\end{tabular}




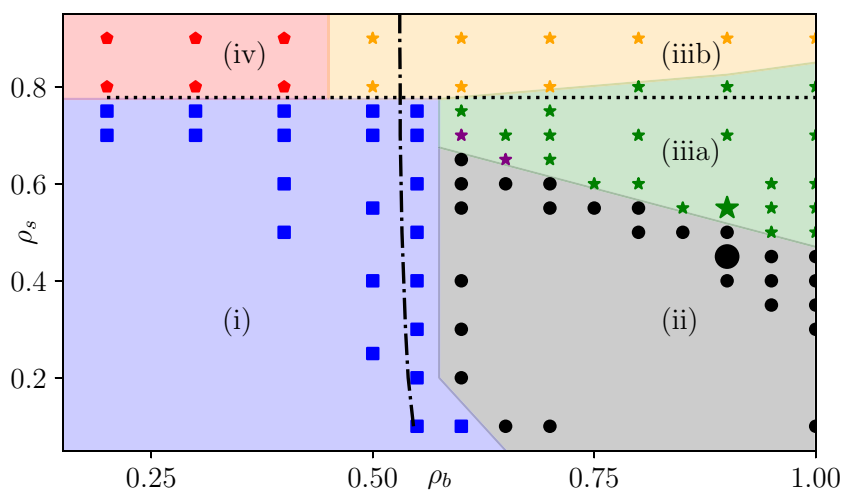

FIG. 1. Generic assembly phase diagram of a two-dimensional (2D) model binary system. Four distinct phases emerge: (i) a phase where both $b$ and $s$ particle densities are spatially uniform (squares); (ii) a phase corresponding to one species condensing into droplets surrounded by the other, i.e., a single-core phase (dots) (results for the large dot shown in Fig. 3); (iii) a multilength scale phase (stars) in which the species assemble into droplets inside droplets with the response corresponding to two subsets: (iiia) multicore micelle-type assembly (results for the large star shown in Fig. 2) where each $s$ island is clearly separate from the other $s$ islands and (iiib) where the $s$ islands overlap at their outskirt regions; and (iv) a phase where the $s$ particles form a hexagonal lattice covering the whole space, while the $b$ particles are homogeneously spread (rhombi). This corresponds to species $b$ being soluble, while $s$ forms droplets. Purple stars are hybrid cases, where most of the islands of $s$ particles behave as the green star states. However, some random clusters do not display the short spacing, and the distribution of the $s$ particles is like the one of the $b$ particles [see Fig. 2(a)]. The dotted line represents the linear instability of the length scale associated with the $s$ particles $k_{s}$, and the dashed-dotted line the one associated with the $b$ particles $k_{b}$. The boundary lines are guides to the eye.

The linear dispersion relation contains all the information required to determine whether or not the uniform liquid is stable with respect to any (small) perturbation. Thus, to resolve thermodynamically stable states where also assemblies with multiple length scales emerge, we address $\omega_{ \pm}(k)$ and look for the crossing point of the two linear instability lines (the dashed and dashed-dotted almost horizontal and vertical lines in Fig. 1). We could have also used Eq. (2) to approximate the crossing point of the two linear instability lines, but $\omega_{ \pm}(k)$ provides the exact information. Unfortunately, knowing this crossing point merely suggests the values of $\rho_{b}$ and $\rho_{s}$ that potentially exhibit equilibrium multiple length scale structure.

The full assembly phase diagram, Fig. 1, based on Eq. (1) and the set of parameters in Table I, is obtained by varying $\rho_{b}$ and $\rho_{s}$. An extensive description of this process is reported in the SM [42]. To facilitate comparison with computer simulations on multicomponent polymer systems in a solvent, we adopt a description of the phase terminology that is specific to polymer self-assembly. The diagram consists of four distinct phases: (i) a phase where both densities are uniform (squares); (ii) a core-shell (or single-core) micelle phase (dots); (iii) a multilength scale phase (stars), composed by two subsets (iiia) and (iiib); and (iv) a phase in which the $s$ particles form a hexagonal structure across the system, while the $b$ particles are homogeneously spread (rhombi). The boundary lines between the different states in Fig. 1 are meant as a guide for the eye and should not be considered as exact.

We start the description of the different phases with subset (iiia), commensurate with multicore-micelle formation in polymer assemblies. An example of this structure is shown in Fig. 2 (large star in Fig. 1). Here, the $b$ particles form a hexagonal structure with lattice spacing of $\approx R_{b b}$, as shown in Fig. 2(a). Simultaneously, the $s$ particles form islands of small hexagonal clusters with lattice spacing of $\approx R_{s s}$, centered at the density maxima of $b$ [panel (b)]. Importantly, the orientations of the $s$ islands are independent of each other at each $b$ maximum. Thus, the $s$ particles are locally ordered within each $b$ maximum only. Such an assembly response describes complex ordered phases in soft matter such as multicore micelles or multiple emulsions. Note that the perfect hexagonal order is due to periodic boundary conditions and lack of thermal fluctuations in the present calculations.

The structural change from (iiia) to (iiib) consists of the $s$ particle islands increasing in size [Fig. 2(b)]. Increasing $\rho_{s}$ eventually bridges the islands and makes them indiscernible. As the $s$ islands differ in orientation, grain boundaries will emerge. We mark the states with at least two merged islands with orange stars. The extreme case is a structure where the $s$ particles fill completely the space (large values of $\rho_{s}$ ).

In contrast, decreasing $\rho_{s}$ starting from states in (iiia) causes the hexagonal arrangement of small islands $s$ to melt into large $s$ droplets centered around the maxima of $\rho_{b}(\mathbf{r})$. However, the attractive nature of $\phi_{b s}$ forces the $s$ particles to remain in the vicinity of such maxima [Fig. 3(b) and inset of Fig. 3(a) show an example corresponding to the large dot in
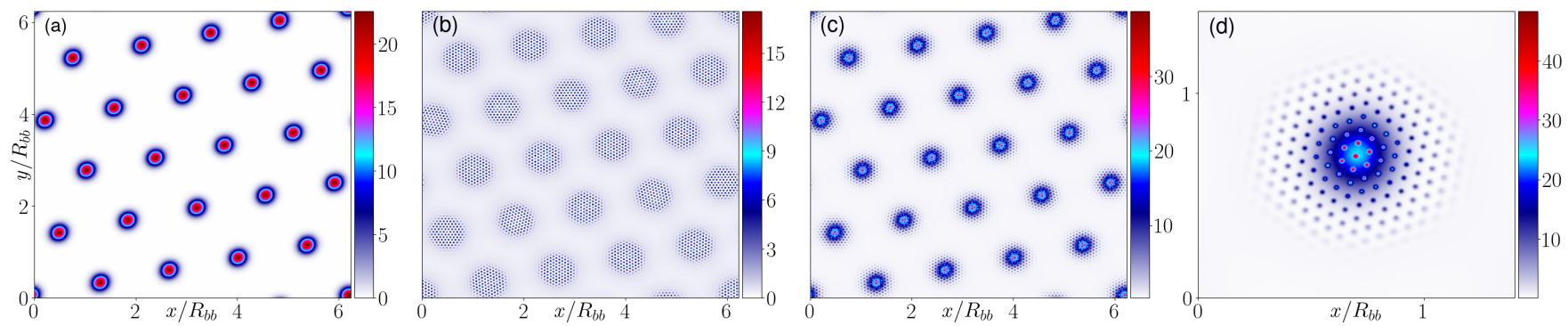

FIG. 2. Dual length scale self-assembled phase (large star in Fig. $1, \rho_{b}=0.9, \rho_{s}=0.55$ ). For polymer systems, this refers to multicore micelles, and for an emulsion to a thermodynamically stable droplet-inside-droplet state (multiple emulsion). (a) shows $\rho_{b}(\mathbf{r})$, (b) $\rho_{s}(\mathbf{r}),(\mathrm{c})$ the total density $\rho_{t}(\mathbf{r})=\rho_{b}(\mathbf{r})+\rho_{s}(\mathbf{r})$, and (d) a blow-up of a single peak. $\rho_{b}(\mathbf{r})$ exhibits hexagonal structure with spacing $\approx R_{b b}$. Cocentric with $\rho_{b}(\mathbf{r})$ peaks, also $\rho_{s}(\mathbf{r})$ forms smaller hexagonal clusters with spacing $\approx R_{s s}$. 


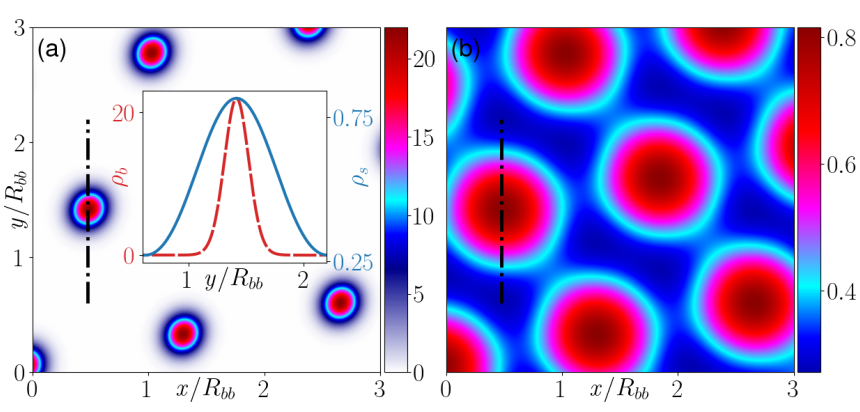

FIG. 3. Single length scale self-assembled phase (large dot in Fig. $1, \rho_{b}=0.9, \rho_{s}=0.45$ ). This corresponds to, e.g., core-shell micelles in polymer systems or a regular emulsion where droplets form into the carrier phase. (a) shows $\rho_{b}(\mathbf{r})$, (b) $\rho_{s}(\mathbf{r})$, and the inset the density profiles calculated along the dash-dotted lines at $x=0.476 R_{b b}$. The solid line represents $\rho_{s}(y)$ and the dashed line the (much larger) density $\rho_{b}(y)$. The system size is $6.22 \times 6.22 R_{b b}^{2}$.

Fig. 1]. This transition corresponds to moving from phase (iii) to phase (ii). The latter structure is commensurate with singlecore (core-shell) micelles. These states survive for decreasing $\rho_{s}$ unless $\rho_{b}<\rho_{b}^{c}$. Figure 1 shows that multicore micelles are obtained mostly for $\rho_{s}<\rho_{s}^{c}$, i.e., for densities at which the system is linearly stable with respect to $k_{s}$. The deviation of the actual phase boundaries from the linear instability line can be explained as follows: consider a single-core micelle state. If the average density of the $s$ particles in each of these peaks is roughly of the same order as $\rho_{s}^{c}$, the $s$ particles find energetically favorable to form clusters with typical spacing of $\approx 2 \pi / k_{s}[(\mathrm{ii}) \rightarrow$ (iii)]. This can be seen as a local instability occurring at the level of a single $b$ site.

The two purple stars in (iiia) represent a state in which most of the $s$ clusters behave as in the case represented by green stars [Fig. 2(b)]. However, some random clusters of $s$ particles do not display the short length scale order, but instead, their distribution is like the one of the $b$ particles [Gaussian-like distributed; Fig. 2(a)]. These states might be metastable due to the vicinity with phase boundaries.

In (iv), the system is filled by a hexagonal structure with spacing of $\approx R_{s s}$. Simultaneously, $\rho_{b}(\mathbf{r})$ is uniform. This phase can be found for $\rho_{s}>\rho_{s}^{c}$ and $\rho_{b}<\rho_{b}^{c}$. The attractive nature of $\phi_{b s}$ favors the orange-star states to exist down to values of $\rho_{b}<\rho_{b}^{c}$, where one would expect to find rhombi states if only linear instabilities were considered. Cluster formation of $b$ particles is enhanced by the attraction mediated by clusters of $s$ particles. This can be explained in a manner similar to the discrepancy between the linear instability line of the $s$ particles and the boundary between dots and stars.

Finally, in phase (i), both species are uniformly distributed, i.e., a solution of miscible species. The slight discrepancy between the linear instability line of particles $b$ and the boundary between the homogeneous state and the states in which the latter species is linearly unstable is due to finite-size effects and to the fact that the dispersion relation only considers the linear contributions of the dynamics. Furthermore, the size of the system has been chosen randomly, which may lead to a wave number slightly different from $k_{b}$. Here, $\omega_{+}\left(k_{b}\right)$ is concave with a maximum at $k_{b}$ at the onset of the instability: particles $b$ will form clusters for a slightly different value
TABLE II. Symmetries of $\rho_{b}$ and $\rho_{s}$ in the different phases.

\begin{tabular}{ccccc}
\hline \hline State & i & ii & iii & iv \\
\hline$\rho_{b}$ & Even & Hex $R_{b b}$ & Hex $R_{b b}$ & Even \\
$\rho_{s}$ & Even & Hex $R_{b b}$ & Hex $R_{s s}$ islands over hex $R_{b b}$ & $\operatorname{Hex~} R_{s s}$ \\
\hline \hline
\end{tabular}

of $\rho_{b}$. The symmetries of $\rho_{b}(\mathbf{r})$ and $\rho_{s}(\mathbf{r})$ in the different phases are summarized in Table II. We note that the transition between phases (ii) and (iii) is discontinuous. This is due to spatial symmetry breaking between these phases.

To compare the emergence of the abovediscussed single vs multiple length scale assembly in a realistic molecular level system, we perform dissipative particle dynamics (DPD) [51-54] simulations of a polymeric solution composed of a solvophobic polymer $\left(A_{19}\right)$ and a diblock copolymer $\left(A_{1} B_{6}\right)$ in a solvent using the LAMMPS [55] simulation package. The segments $A$ and $B$ are solvophobic and solvophilic, respectively. The subscripts refer to the number of DPD beads (block lengths). Each bead represents a group of atoms which experience a force $\mathbf{F}_{i}=\sum_{j \neq i} \mathbf{F}_{i j}^{C}+\mathbf{F}_{i j}^{D}+\mathbf{F}_{i j}^{R}, i, j=1, \cdots, N$, where $\mathbf{F}^{C}$ describes conservative interactions, $\mathbf{F}^{D}$ dissipative contributions, $\mathbf{F}^{R}$ random contribution, and where $N$ is the total number of beads. The interaction forces are treated as pairwise additive and are truncated at a distance $r_{c}$. In the polymer chains, adjacent beads also contribute to a springlike force $\mathbf{F}^{S}$. These soft potentials facilitate acceleration of the numerical simulations so that realistic experimental time and length scales can be achieved. Further details and simulation specifics can be found in the SM [42].

Figure 4 shows snapshots of different equilibrium configurations obtained from simulations by varying the molar fractions of the components. This system strikingly selfassembles into structures corresponding to multicore and single-core corona states. Both cases correspond to a total solid content of $N_{s} /\left(N_{s}+N_{w}\right)=20.15 \%$ in aqueous solvent,

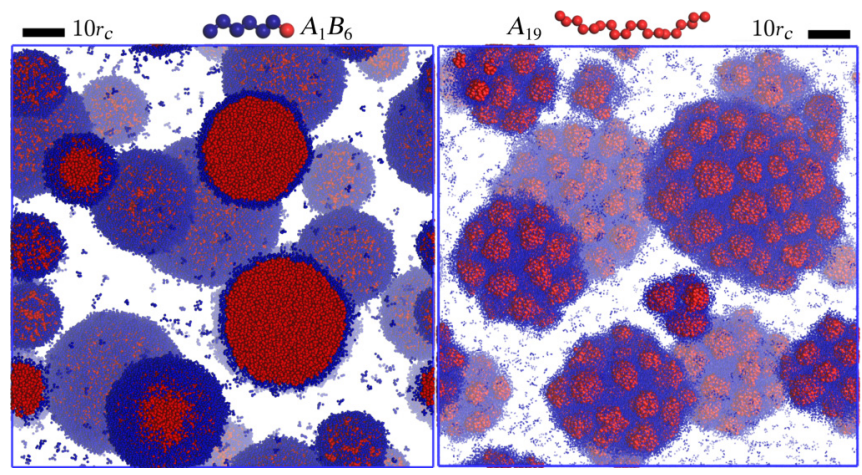

FIG. 4. Equilibrium configurations from three-dimensional (3D) dissipative particle dynamics (DPD) simulations of a polymeric solution composed of a solvophobic polymer $\left(A_{19}\right)$ and a diblock copolymer $\left(A_{1} B_{6}\right)$ in a solvent. The segments $A$ (red) and $B$ (blue) are solvophobic and solvophilic, respectively. The subscript refers to the number of DPD beads. Left: single-core (core-shell) configuration for molar fractions $50 \% A_{19}$ and $50 \% A_{1} B_{6}$. Right: multicore configuration for molar fractions $10 \% A_{19}$ and $90 \% A_{1} B_{6}$. The solvent is not shown for clarity. 
where $N_{s}$ and $N_{w}$ are the number of solid and water beads, respectively. In the former case, the molar fractions are $10 \%$ $A_{19}$ and $90 \% A_{1} B_{6}$, while in the latter, $50 \% A_{19}$ and $50 \% A_{1} B_{6}$. The simulations are performed in a cubic box of $100 \times 100 \times$ $100 r_{c}^{3}$. These equilibrium configurations show dual and single length scale structural assemblies analogous to those in our DFT based phase diagram [phases (ii) and (iiia) in Fig. 1]. It is tempting to interpret a DPD polymer chain as a single coarse-grained DFT particle, but it should be noted that the surfactantlike nature of the copolymer makes a quantitative comparison between chemically predictive molecular model and DFT-assembly structures difficult. Nevertheless, the two distinct length scales emerging from the effective interactions in Fig. 2 also appear in the DPD model. Additionally, in DPD simulations, the key feature for multicore assembly is a sufficient degree of immiscibility between the species. The formation of solvophobic cores needs to be energetically favorable and sufficiently stabilized by the solvophilic polymer segments, and the solvophilic polymer cannot have too favorable interactions with either the solvent or the solvophobic polymer. If either the solvophobic polymers are too solvophobic, the solvophilic one too solvophilic, or the two polymers readily mix, the assembly becomes core-shell or phase separates. This demonstrates how competition between interactions and mutual balance of immiscibility gives rise to multiple length scale assemblies-single length scale selfassembly is retained when any of the pairwise effective interactions dominates.

To summarize, we have presented a microscopic theory capable of explaining self-assembly within soft matter with multiple competing length scales. The theory relies on the interplay between the effective interaction potentials modeling the different constituents of the system. The significance of our findings resides in the identification of key molecular features involved in general multiple length scale self-assembly. Although multiple length scale assembly is ubiquitous, such guidelines have been lacking until now. By providing much needed insight to understanding, e.g., morphologies rising in intracellular biocondensates, multiple emulsions, or lipid bilayer microdomains, we obtain means to engineer and tune soft matter to desired multiple length scale structures. In addition to obvious applications to multiple length scale self-assembling compartmentalization, such as drug delivery, catalysis, and selective multistep reaction platforms, our work provides tools for harnessing the full potential of revolutionary materials production via biological mechanisms, advanced engineered biomaterials, or complex polymer assemblies, providing crucial insight on how to tune the assembly response.

A.S. is thankful to A. J. Archer for fruitful discussions. This paper was supported by Academy of Finland Grant No. 309324 (M.S.) and No. 307806 and No. 312298 (T.A.-N.). T.A.-N. has also been supported by a Technology Industries of Finland Centennial Foundation TT2020 grant. We are grateful for the support by FinnCERES Materials Bioeconomy Ecosystem. Computational resources by CSC IT Centre for Finland and RAMI-RawMatters Finland Infrastructure are also gratefully acknowledged.

This paper was conceived and supervised by T.A.-N. and M.S. A.S. formulated and executed the theoretical calculations and wrote the first draft of the manuscript. S.J.N. carried out the molecular simulations. All authors contributed to writing.
[1] B. J. G. E. Pieters, M. B. van Eldijk, R. J. M. Nolte, and J. Mecinović, Natural supramolecular protein assemblies, Chem. Soc. Rev. 45, 24 (2016).

[2] S. F. Banani, H. O. Lee, A. A. Hyman, and M. K. Rosen, Biomolecular condensates: organizers of cellular biochemistry, Nat. Rev. Mol. Cell Biol. 18, 285 (2017).

[3] R. Zandi, B. Dragnea, A. Travesset, and R. Podgornik, On virus growth and form, Phys. Rep. 847, 1 (2020).

[4] E. Sezgin, I. Levental, S. Mayor, and C. Eggeling, The mystery of membrane organization: composition, regulation and roles of lipid rafts, Nat. Rev. Mol. Cell Biol. 18, 361 (2017).

[5] M. A. Meyers, P.-Y. Chen, A. Y.-M. Lin, and Y. Seki, Biological materials: Structure and mechanical properties, Prog. Mater. Sci. 53, 1 (2008).

[6] M. A. Cohen Stuart, W. T. Huck, J. Genzer, M. Müller, C. Ober, M. Stamm, G. B. Sukhorukov, I. Szleifer, V. V. Tsukruk, M. Urban, F. Winnik, S. Zauscher, I. Luzinov, and S. Minko, Emerging applications of stimuli-responsive polymer materials, Nat. Mater. 9, 101 (2010).

[7] L. MacFarlane, H. Shaikh, J. Garcia-Hernandez, M. Vespa, T. Fukui, and I. Manners, Functional nanoparticles through $\pi$ conjugated polymer self-assembly, Nat. Rev. Mater. 6, 7 (2021).

[8] Y. Lu, J. Lin, L. Wang, L. Zhang, and C. Cai, Self-assembly of copolymer micelles: higher-level assembly for constructing hierarchical structure, Chem. Rev. 120, 4111 (2020).
[9] I. I. Smalyukh, Liquid crystal colloids, Annu. Rev. Condens. Matter Phys. 9, 207 (2018).

[10] K. R. Phillips, G. T. England, S. Sunny, E. Shirman, T. Shirman, N. Vogel, and J. Aizenberg, A colloidoscope of colloid-based porous materials and their uses, Chem. Soc. Rev. 45, 281 (2016).

[11] C. Gong, S. Sun, Y. Zhang, L. Sun, Z. Su, A. Wu, and G. Wei, Hierarchical nanomaterials via biomolecular selfassembly and bioinspiration for energy and environmental applications, Nanoscale 11, 4147 (2019).

[12] Y. Wang, J. Guo, L. Zhou, C. Ye, F. G. Omenetto, D. L. Kaplan, and S. Ling, Design, fabrication, and function of silk-based nanomaterials, Adv. Funct. Mater. 28, 1805305 (2018).

[13] T. Lu and E. Spruijt, Multiphase complex coacervate droplets, J. Am. Chem. Soc. 142, 2905 (2020).

[14] T. Sheth, S. Seshadri, T. Prileszky, and M. E. Helgeson, Multiple nanoemulsions, Nat. Rev. Mater. 5, 214 (2020).

[15] L.-L. Ma, W. Hu, Z.-G. Zheng, S.-B. Wu, P. Chen, Q. Li, and Y.-Q. Lu, Light-activated liquid crystalline hierarchical architecture toward photonics, Adv. Opt. Mater. 7, 1900393 (2019).

[16] M. Qi and Y. Zhou, Multimicelle aggregate mechanism for spherical multimolecular micelles: from theories, characteristics and properties to applications, Mater. Chem. Front. 3, 1994 (2019). 
[17] S. Casalini, C. A. Bortolotti, F. Leonardi, and F. Biscarini, Selfassembled monolayers in organic electronics, Chem. Soc. Rev. 46, 40 (2017).

[18] M. York-Duran, M. Godoy-Gallardo, C. Labay, A. Urquhart, T. Andresen, and L. Hosta-Rigau, Recent advances in compartmentalized synthetic architectures as drug carriers, cell mimics and artificial organelles, Colloids Surf. B: Biointerfaces 152, 199 (2017).

[19] J. Gaitzsch, X. Huang, and B. Voit, Engineering functional polymer capsules toward smart nanoreactors, Chem. Rev. 116, 1053 (2016).

[20] M. S. Aw, M. Kurian, and D. Losic, Polymeric micelles for multidrug delivery and combination therapy, Chem. Eur. J. 19, 12586 (2013).

[21] M. Wu, Y. Zhu, and W. Jiang, Disassembly of multicompartment polymer micelles in spatial sequence using an electrostatic field and its application for release in chronological order, Angew. Chem., Int. Ed. 57, 3578 (2018).

[22] R. Nagarajan and E. Ruckenstein, Theory of surfactant selfassembly: A predictive molecular thermodynamic approach, Langmuir 7, 2934 (1991).

[23] V. Palermo and P. Samorí, Molecular self-assembly across multiple length scales, Angew. Chem., Int. Ed. 46, 4428 (2007).

[24] Theory of Simple Liquids, fourth ed., edited by J.-P. Hansen and I. R. McDonald (Academic Press, Oxford, 2013).

[25] R. Evans, The nature of the liquid-vapour interface and other topics in the statistical mechanics of non-uniform, classical fluids, Adv. Phys. 28, 143 (1979).

[26] R. Evans, Density functionals in the theory of non-uniform fluids, in Fundamentals of Inhomogeneous Fluids, edited by D. Henderson (Dekker, New York, 1992), pp. 85-175.

[27] J. F. Lutsko, Recent developments in classical density functional theory, Adv. Chem. Phys. 144, 1 (2010).

[28] H. Chen and E. Ruckenstein, Formation and degradation of multicomponent multicore micelles: insights from dissipative particle dynamics simulations, Langmuir 29, 5428 (2013).

[29] N. Duxin, F. Liu, H. Vali, and A. Eisenberg, Cadmium sulphide quantum dots in morphologically tunable triblock copolymer aggregates, J. Am. Chem. Soc. 127, 10063 (2005).

[30] M. Ueda, A. Hashidzume, and T. Sato, Unicore- multicore transition of the micelle formed by an amphiphilic alternating copolymer in aqueous media by changing molecular weight, Macromolecules 44, 2970 (2011).

[31] H. Chen and E. Ruckenstein, Formation of complex colloidal particles: Morphologies and mechanisms, Soft Matter 8, 8911 (2012).

[32] P. Bolhuis, A. Louis, J. Hansen, and E. Meijer, Accurate effective pair potentials for polymer solutions, J. Chem. Phys. 114, 4296 (2001).

[33] C. N. Likos, Effective interactions in soft condensed matter physics, Phys. Rep. 348, 267 (2001).

[34] C. Likos, H. Löwen, M. Watzlawek, B. Abbas, O. Jucknischke, J. Allgaier, and D. Richter, Star Polymers Viewed as Ultrasoft Colloidal Particles, Phys. Rev. Lett. 80, 4450 (1998).

[35] A. Louis, P. Bolhuis, J. Hansen, and E. Meijer, Can Polymer Coils be Modeled as "Soft Colloids"? Phys. Rev. Lett. 85, 2522 (2000).
[36] I. Götze, H. Harreis, and C. Likos, Tunable effective interactions between dendritic macromolecules, J. Chem. Phys. 120, 7761 (2004).

[37] C. N. Likos, Soft matter with soft particles, Soft Matter 2, 478 (2006).

[38] D. A. Lenz, R. Blaak, C. N. Likos, and B. M. Mladek, Microscopically Resolved Simulations Prove the Existence of Soft Cluster Crystals, Phys. Rev. Lett. 109, 228301 (2012).

[39] B. M. Mladek, D. Gottwald, G. Kahl, M. Neumann, and C. N. Likos, Formation of Polymorphic Cluster Phases for a Class of Models of Purely Repulsive Soft Spheres, Phys. Rev. Lett. 96, 045701 (2006).

[40] C. N. Likos, B. M. Mladek, D. Gottwald, and G. Kahl, Why do ultrasoft repulsive particles cluster and crystallize? Analytical results from density-functional theory, J. Chem. Phys. 126, 224502 (2007).

[41] T. V. Ramakrishnan and M. Yussouff, First-principles orderparameter theory of freezing, Phys. Rev. B 19, 2775 (1979).

[42] See Supplemental Material at http://link.aps.org/supplemental/ 10.1103/PhysRevResearch.3.L022008 for details on density functional theory and dissipative particle dynamics simulations.

[43] W. Somerville, J. Stokes, A. Adawi, T. Horozov, A. J. Archer, and D. Buzza, Density functional theory for the crystallization of two-dimensional dipolar colloidal alloys, J. Phys.: Condens. Matter 30, 405102 (2018).

[44] A. Scacchi, W. Somerville, D. Buzza, and A. Archer, Quasicrystal formation in binary soft matter mixtures, Phys. Rev. Research 2, 032043 (2020).

[45] A. J. Archer, M. C. Walters, U. Thiele, and E. Knobloch, Solidification in soft-core fluids: disordered solids from fast solidification fronts, Phys. Rev. E 90, 042404 (2014).

[46] A. J. Archer and R. Evans, Dynamical density functional theory and its application to spinodal decomposition, J. Chem. Phys. 121, 4246 (2004).

[47] A. J. Archer, Dynamical density functional theory for dense atomic liquids, J. Phys.: Condens. Matter 18, 5617 (2006).

[48] A. J. Archer, A. M. Rucklidge, and E. Knobloch, Quasicrystalline Order and a Crystal-Liquid State in a Soft-Core Fluid, Phys. Rev. Lett. 111, 165501 (2013).

[49] A. J. Archer, A. M. Rucklidge, and E. Knobloch, Soft-core particles freezing to form a quasicrystal and a crystal-liquid phase, Phys. Rev. E 92, 012324 (2015).

[50] M. C. Walters, P. Subramanian, A. J. Archer, and R. Evans, Structural crossover in a model fluid exhibiting two length scales: repercussions for quasicrystal formation, Phys. Rev. E 98, 012606 (2018).

[51] P. Hoogerbrugge and J. Koelman, Simulating microscopic hydrodynamic phenomena with dissipative particle dynamics, Europhys. Lett. 19, 155 (1992).

[52] P. Español and P. Warren, Statistical mechanics of dissipative particle dynamics, Europhys. Lett. 30, 191 (1995).

[53] R. D. Groot and P. B. Warren, Dissipative particle dynamics: bridging the gap between atomistic and mesoscopic simulation, J. Chem. Phys. 107, 4423 (1997).

[54] P. Español and P. B. Warren, Perspective: dissipative particle dynamics, J. Chem. Phys. 146, 150901 (2017).

[55] S. Plimpton, Fast parallel algorithms for short-range molecular dynamics, J. Comput. Phys. 117, 1 (1995). 